\title{
Multidisciplinary analysis of the Hapi region located on Comet 67P/Churyumov-Gerasimenko
}

\author{
M. Pajola ${ }^{\oplus},{ }^{\star}{ }$ J.-C. Lee, ${ }^{2 \star}$ N. Oklay, ${ }^{3}$ S. F. Hviid, ${ }^{3}$ L. Penasa ${ }^{\oplus},{ }^{4}$ S. Mottola, ${ }^{3}$ X. Shi, ${ }^{5}$ \\ S. Fornasier ${ }^{\oplus}{ }^{6}$ B. Davidsson, ${ }^{7}$ L. Giacomini ${ }^{8}$ A. Lucchetti ${ }^{\oplus},{ }^{1}$ M. Massironi, ${ }^{9}$ \\ J. B. Vincent ${ }^{\oplus},{ }^{3}$ I. Bertini, ${ }^{10}$ G. Naletto ${ }^{\oplus}, 4,10,11$ W. H. Ip, ${ }^{12,13}$ H. Sierks, ${ }^{5}$ P. L. Lamy, ${ }^{14}$ \\ R. Rodrigo, ${ }^{15,16}$ D. Koschny, ${ }^{17}$ H. U. Keller, ${ }^{3,18}$ J. Agarwal, ${ }^{5}$ M. A. Barucci ${ }^{\bullet}, 6$ \\ J. L. Bertaux ${ }^{\odot},{ }^{14}$ D. Bodewits, ${ }^{19}$ P. Cambianica, ${ }^{4}$ G. Cremonese,${ }^{1}$ V. Da Deppo,${ }^{11}$ \\ S. Debei, ${ }^{20}$ M. De Cecco, ${ }^{21}$ J. Deller ${ }^{\circ},{ }^{5}$ M. R. El Maarry ${ }^{22}$ C. Feller, ${ }^{6}$ S. Ferrari, ${ }^{4}$ \\ M. Fulle ${ }^{\oplus},{ }^{23}$ P. J. Gutierrez, ${ }^{24}$ C. Güttler ${ }^{\oplus},{ }^{5}$ L. M. Lara, ${ }^{24}$ F. La Forgia, ${ }^{10}$ \\ M. Lazzarin, ${ }^{10}$ Z.-Y. Lin, ${ }^{12}$ J. J. Lopez Moreno, ${ }^{24}$ F. Marzari, ${ }^{10}$ F. Preusker, ${ }^{3}$ \\ F. Scholten, ${ }^{3}$ I. Toth ${ }^{25}$ and C. Tubiana ${ }^{\oplus 5}$ \\ Affiliations are listed at the end of the paper
}

Accepted 2019 February 6. Received 2019 January 28; in original form 2018 August 10

\begin{abstract}
By using the Rosetta/OSIRIS-NAC data set taken in 2014 August, we focus on the neck region, called Hapi, located on 67P Churyumov-Gerasimenko's Northern hemisphere. The gravitational potential and slopes of Hapi, coupled with the geological unit identification and the boulder size-frequency distributions, support the interpretation that both taluses and gravitational accumulation deposits observable on Hapi are the result of multiple cliff collapses that occurred at different times. By contrast, the fine-particle deposits observable in the central part of the study area are made of aggregates coming from the Southern hemisphere and deposited during each perihelion passage. Both the consolidated terrains on the western part of Hapi, as well as the centrally aligned ridge made of boulder-like features, suggest that Hapi is in structural continuity with the onion-like structure of the main lobe of 67P. Despite the dusty blanket observable on Hapi, its terrains are characterized by water-ice-rich components that, once repeatedly and rapidly illuminated, sublimate, hence resulting in the strong jet activity observed in 2014 August.
\end{abstract}

Key words: methods: data analysis - methods: statistical - comets: individual (67P C-G).

\section{INTRODUCTION}

On 2014 August 6, the European Space Agency's Rosetta spacecraft started orbiting the Jupiter-family comet 67P/ChuryumovGerasimenko (hereafter 67P). Since then, and until Rosetta lands on the surface of 67P, a wealth of high-resolution images have been taken by the OSIRIS instrument (Optical, Spectroscopic and Infrared Remote Imaging System, Keller et al. 2007), returning some of the most detailed pictures of a cometary surface thus far. The analysis of the first OSIRIS images highlighted that 67P is a bi-lobed nucleus (Sierks et al. 2015) characterized by a morphologically

\footnotetext{
*E-mail: maurizio.pajola@inaf.it, maurizio.pajola@gmail.com (MP),
} vickielee333@gmail.com (JCL) diverse surface despite its small dimensions $(\sim 4 \mathrm{~km}$ size, Thomas et al. 2015a) where active pits (Vincent et al. 2015), ubiquitous boulders (Pajola et al. 2015), highly reflective boulder clusters (Pommerol et al. 2015), fractures (El-Maarry et al. 2015a), dustcovered terrains (Thomas et al. 2015b) and strata (Massironi et al. 2015) are present.

One of the most interesting region of $67 \mathrm{P}$ is the Hapi region, which is the connecting area located between the big and small lobes (called the body and the head, respectively, El-Maarry et al. 2015 b) in close proximity to the North pole of 67P (Preusker et al. 2015). Among the many 67P discoveries that surprised the scientific community was the activity coming from Hapi itself (Sierks et al. (2015). This activity was somehow unexpected because by the time Rosetta rendezvoused with 67P the comet was still at a distance from the Sun $(3.61 \mathrm{au})$ where the nucleus was expected to be still dormant 
Table 1. The OSIRIS-NAC data sets used for the spectrophotometric analysis (row 1). For this analysis the reference image is indicated in bold. Rows 2 and 3 indicate the two images used for the geological and boulder analyses.

\begin{tabular}{|c|c|c|c|c|c|c|}
\hline $\begin{array}{l}\text { Acquisition } \\
\text { date }\end{array}$ & UT & $\begin{array}{l}\text { Filter wavelengths } \\
\qquad(\mathrm{nm})\end{array}$ & $\begin{array}{c}r \\
(\mathrm{au})\end{array}$ & $\begin{array}{l}\text { Distance to 67P } \\
\qquad(\mathrm{km})\end{array}$ & $\begin{array}{c}\text { Scale } \\
\left(\mathrm{mpx}^{-1}\right)\end{array}$ & $\begin{array}{c}\text { Phase } \\
\text { angle }\left({ }^{\circ}\right)\end{array}$ \\
\hline 2014-08-22 & 01:43:49 & $360.0,480.7,535.7, \mathbf{6 4 9 . 2}, 701.2,743.7,805.3,882.1,989.3$ & 3.50 & 65.2 & 1.24 & 34.82 \\
\hline 2014-08-29 & $21: 42: 53$ & 649.2 & 3.45 & 51.3 & 0.91 & 39.52 \\
\hline
\end{tabular}

or characterized by very low activity. Moreover, after computing the energy incident on the entire nucleus over one rotation, ${ }^{1}$ it became clear that the active Hapi region was receiving less energy than other parts of the nucleus that showed little to no sign of surface activity (Sierks et al. 2015). The observed Hapi activity was explained by taking into account self-heating (reflected light and IR radiation) reradiating both from the head and the body, hence providing extra heating (10 per cent higher flux than other regions) to the region of the neck at the time of peak jet activity (Sierks et al. 2015; Keller et al. 2015b).

Between 2014 August and October, the Rosetta spacecraft was placed at distances ranging from 100 to $25 \mathrm{~km}$ from the nucleus, resulting in an almost complete coverage of the northern and equatorial regions of $67 \mathrm{P}$ with a spatial resolution spanning 2 to $0.5 \mathrm{~m}$. By means of this high-resolution image data set, different areas on 67P have been studied in great detail, such as the Maftet, Ma'at, Nut and Hatmehit areas (La Forgia et al. 2015), the Abydos region (Lucchetti et al. 2016), the circular niches in the Seth region (Lucchetti et al. 2017), the Aswan site in the Seth region (Pajola et al. 2016d) and the Imhotep region (Auger et al. 2015). These analyses focused on localized areas of interest on 67P, expanding upon the geological maps presented in Giacomini et al. (2016). In addition, the mentioned works added gravitational slope maps, boulder sizefrequency distributions and colour maps to the geological units, providing an increasingly detailed picture of the nucleus of $67 \mathrm{P}$ before its perihelion.

Such a multidisciplinary approach has not yet been done for the Hapi region before; hence, it is accomplished here to increase the coverage of 67P's pre-perihelion surface analysis and to facilitate the detection of subsequent surface changes.

This paper is structured as follows: after the description of the OSIRIS data set and methods, we focus on the location and gravitational framework of Hapi, detailing its geological units and the boulder size-frequency distributions derived on the entire study area, as well as on specific geological units. We present the evidence localized on Hapi that further supports the layered structure of comet 67P. We focus on the colours and spectrophotometry of the studied terrains, and then on the peculiar thermal behaviour of the neck region. Finally, we discuss the jet activity departing from Hapi, contextualizing it with the comet's global activity.

\section{DATA SET AND METHODS}

Our multidisciplinary analysis of the Hapi region was performed using OSIRIS-Narrow Angle Camera (NAC) data sets taken on 2014 August 22 and 29, respectively. The acquisition dates, filters used, observation geometries, scales and phase angles are summarized in Table 1. The images belonging to the first data set (row 1 of Table 1) have a spatial scale ranging from 1.14 to $1.24 \mathrm{~m} \mathrm{pixel}^{-1}$; they were taken on August 22 with a phase angle of $\sim 35^{\circ}$ and were specifically used for the spectrophotometric analysis. The peculiar location of $\mathrm{Hapi}^{2}$ did not allow full views of the entire area with multiple filters. The selected imagery therefore provides the best compromise between spatial scale and multiband coverage within the entire OSIRIS-NAC pre-perihelion data set. The two images taken on August 29 present a smaller scale of 0.91-0.95 $\mathrm{m} \mathrm{pixel}^{-1}$; they were taken with a phase angle of $\sim 38-40^{\circ}$ and fully cover the Hapi region with only partial shadows. Hence, they were used to prepare the geological map of Hapi and to identify all boulders $(\geq 4.0 \mathrm{~m})$ located in the area.

To describe the gravitational framework of the Hapi region we made use of the stereo-photogrammetric model (SPG) of the comet (Preusker et al. 2017), which has a spatial resolution of $1.5 \mathrm{~m}$. We then derived the gravitational potential of this highly irregular comet, assuming a homogeneous nucleus with a density of $538 \mathrm{~kg} \mathrm{~m}^{-3}$ (Preusker et al. 2017) following the theory of Werner \& Scheeres (1997) and accounting for the centrifugal force due to the comet rotation (Rossi \& Fulchignoni 1999). We consequently derived the effective gravity on 67P and the gravitational slope map, defined as the angle between the local surface normal pointing inside the nucleus and the gravity vector.

To identify the geological units and linear features present on Hapi we used the same criteria that were used to map the Northern (Giacomini et al. 2016) and Southern hemispheres of 67P (Lee et al. 2016). Hence, geological units characterized by similar texture and morphology, stratigraphic position and possibly source region are grouped into the same class (Birch et al. 2017). Linear features with similar origins are grouped into the same class too. The identification of the unit boundaries was supported by wide use of the high-resolution 3D shape model of the region as well. Then, in order to draw the units and the linear features of Hapi we used the ESRI ARCGIS 10.2 software.

The identification of the boulders located on the different units of Hapi was also done with the ARCGIS 10.2 software. We set the lowest measurable boulder size at $4.0 \mathrm{~m}$ because below this value the distribution starts to roll over, indicating that the size-frequency distribution (SFD) might not be complete (as indicated for example in Michikami et al. 2008; Pajola et al. 2016b). In addition, the $4.0 \mathrm{~m}$ limit value returns a meaningful size-frequency statistics because it is one pixel larger than the three-pixel sampling (Pajola et al. 2016d), hence minimizing the likelihood of misidentifications. Given that the observations were performed with a phase angle of $\sim 40^{\circ}$, the elongated shadows on the surface offered the possibility of identifying boulders that are smaller than four pixels in diameter (two to three pixels in diameter, 2.0-3.0 $\mathrm{m}$ in size); however, we excluded these smaller boulders in the cumulative size-frequency

${ }^{2}$ Hapi is located between the two lobes, which cast mutual shadows multiple times during a cometary day.

${ }^{1}$ This was computed for the 2014 August period, (Sierks et al. 2015). 
distribution because they do not represent a complete data set for such small sizes (Mazrouei et al. 2014; Pajola et al. 2017b).

As in previous works (Pajola et al. 2015, 2016c), we considered a 'boulder' to be a positive relief feature that appears to be detached from the ground on which it stands, that is detectable in different images obtained with various observation geometries and that is characterized by an elongated shadow (if the phase angle is greater than $0^{\circ}$ ). On Hapi there are specific areas where fine-particle deposits partially bury and embed positive features. For this reason the identification of boulders is here made more difficult with respect to other locations on 67P. None the less, the geological context for the identified deposits supports the interpretation that their origin may be related to different cliff collapses; therefore they can still be considered boulders. After manually identifying all boulders in the high-resolution images, we measured their positions on the surface of the comet, assumed their shapes to be circumcircles, and computed their diameters and the corresponding projected areas. In order to obtain the cumulative boulder size-frequency distribution per square kilometre we used the corresponding area computed from the 3D shape model of Hapi. Subsequently, in all log-log plots we fitted a regression line to the data to derive the power-law index of each size distribution, while the error bars for each value indicate the root of the cumulative number of counting boulders, following Michikami et al. (2008) and Pajola et al. (2016a).

To generate a geological cross-section that highlights the orientation of the layers in Hapi and in its closest surroundings, we made use of the Penasa et al. (2017) 3D layered model. This model considers the two lobes of 67P as two individual objects with an onion-like layering joined through the neck region. To understand if some of the geological features identified on Hapi are the expression of the inner layered structure, we mapped structurally coherent outcropping terrains whose geometry was then compared with the model's predictions, hence constraining the structural pertinence of the Hapi terrains to either lobe.

Multispectral images taken at different wavelengths (Table 1, row 1) are co-registered to a reference (bold in Table 1) using the Integrated Software for Imagers and Spectrometers (USGS ISIS $3,{ }^{3}$ Anderson et al. 2004) at sub-pixel accuracy to eliminate the mismatch between images resulting from the rotation of the comet and the motion of spacecraft during imaging of the colour sequence. Illumination variations between images are corrected by dividing reflectance images (I/F) by the Lommel-Seeliger disc function (Hapke 1993). The photometric angles are calculated using the full-resolution SPG SHAP7 model (Preusker et al. 2017). The calculation of the photometric angles at the time of the observations is performed by using SPICE kernels. ${ }^{4}$ The image registration, photometric correction and the generation of the spectral products are achieved with the pipeline described in Oklay et al. (2016).

In order to directly compare the regions observed at different times under different viewing geometries and spatial resolutions, all spectral products are projected on to the 3D shape of comet 67P (Preusker et al. 2017, decimated to $2 \cdot 10^{5}$ facets) using the SHAPEVIEWER software, ${ }^{5}$ and then displayed in the frame-ofreference images.

\footnotetext{
${ }^{3}$ http://isis.astrogeology.usgs.gov/index.html

${ }^{4} \mathrm{ftp}$ ://psa.esac.esa.int/pub/mirror/INTERNATIONAL-ROSETTA-MISS ION/SPICE/RO-RL-E-M-A-C-SPICE-6-V1.0/

${ }^{5}$ http://www.comet-toolbox.com/shapeViewer.html
}

In order to detect variegation on Hapi, spectral slopes are calculated using:

Spectral slope [per cent $\left.100 \mathrm{~nm}^{-1}\right]=\frac{\left(R_{882.1}-R_{535.7}\right) \times 10^{4}}{(882.1-535.7) \times R_{535.7}},(1)$

where $\mathrm{R}_{i}$ is the reflectance computed at the wavelength $i$. Regions of interest (ROIs) are then identified on Hapi to investigate the spectral properties of different geological units. Mean spectral profiles are calculated within each ROI larger than 3000 pixels and then normalized at $480.7 \mathrm{~nm}$ for direct spectral comparisons.

Subsequently, in order to study the thermal behaviour of the Hapi region and evaluate how the surface temperatures changed in the 2014 August timeframe, we applied two Keller et al. (2015b) models and compared Hapi's trends with four reference areas on 67P: Hatmehit and Ma'at on the head, Imhotep and Ash on the body. Specifically, we applied model B (characterized by a $50 \mu \mathrm{m}$ thick layer of dust above the ice/dust mixture of the nucleus) and model C (characterized by a $1 \mathrm{~mm}$ layer above the ice/dust mixture of the nucleus).

\section{MULTIDISCIPLINARY ANALYSES: RESULTS}

\subsection{Location and gravitational framework}

The Hapi region has an area of $1.18 \mathrm{~km}^{2}$; it is located on the neck of 67P close to its North pole (El-Maarry et al. 2015b). Towards the head it is limited by the $900 \mathrm{~m}$ high Hathor cliff that rises perpendicular to the comet's equatorial plane (Fig. 1). The Seth region, characterized by alternating sets of terraces and steep walls, forms the boundary towards the body. To the west, Hapi is bordered by the consolidated Anuket region, while to the east it is bordered by the transitional region Babi and the consolidated Aker region (El-Maarry et al. 2015b).

Hapi is a gravitational low with gravitational potential values reaching the minimum amount on 67P,$-0.49 \mathrm{Nm} \mathrm{kg}^{-1}$ (Figs $2 \mathrm{a}$ and b). The effective gravity on Hapi is minimum in close proximity to the Hathor cliff, while it reaches a value of $0.1 \mathrm{~mm} \mathrm{~s}^{-2}$ towards the Seth region (Figs 2c and d). The computed dynamic height for Hapi, which is the distance from the centre of mass that would produce the gravitational potential assuming that $67 \mathrm{P}$ is a point mass, ranges from 100 to $300 \mathrm{~m}$ (Figs 2e and f). The gravitational slope map indicates that, with the exception of some outcrops located in the middle of Hapi, the central part of the studied area is almost completely flat (values of $2-4^{\circ}$ ), while it rapidly increases towards the bordering regions (Figs $2 \mathrm{~g}$ and $\mathrm{h}$ ).

\subsection{Geological map}

By following the same geological interpretation texture criteria presented in Giacomini et al. (2016) and Lee et al. (2016), we have identified on Hapi three different linear features: strata, terraces/cuestas and fractures (Fig. 3), and six different geological units: 1) fine-particle deposits with or 2) without boulders, 3) taluses, 4) gravitational accumulation deposits, 5) diamictons and 6) outcropping consolidated terrains (Fig. 3). We follow the definition of geological units from Lee et al. (2016), hence dividing our units into consolidated material and non-cohesive deposits. For the Hapi case, the outcropping consolidated terrain is the only geological unit that belongs to the consolidated material class. All other geological units are non-cohesive deposits. We can further divide the noncohensive deposits into mass-wasting deposits and fine material. Within mass-wasting deposits there are then three units defined 


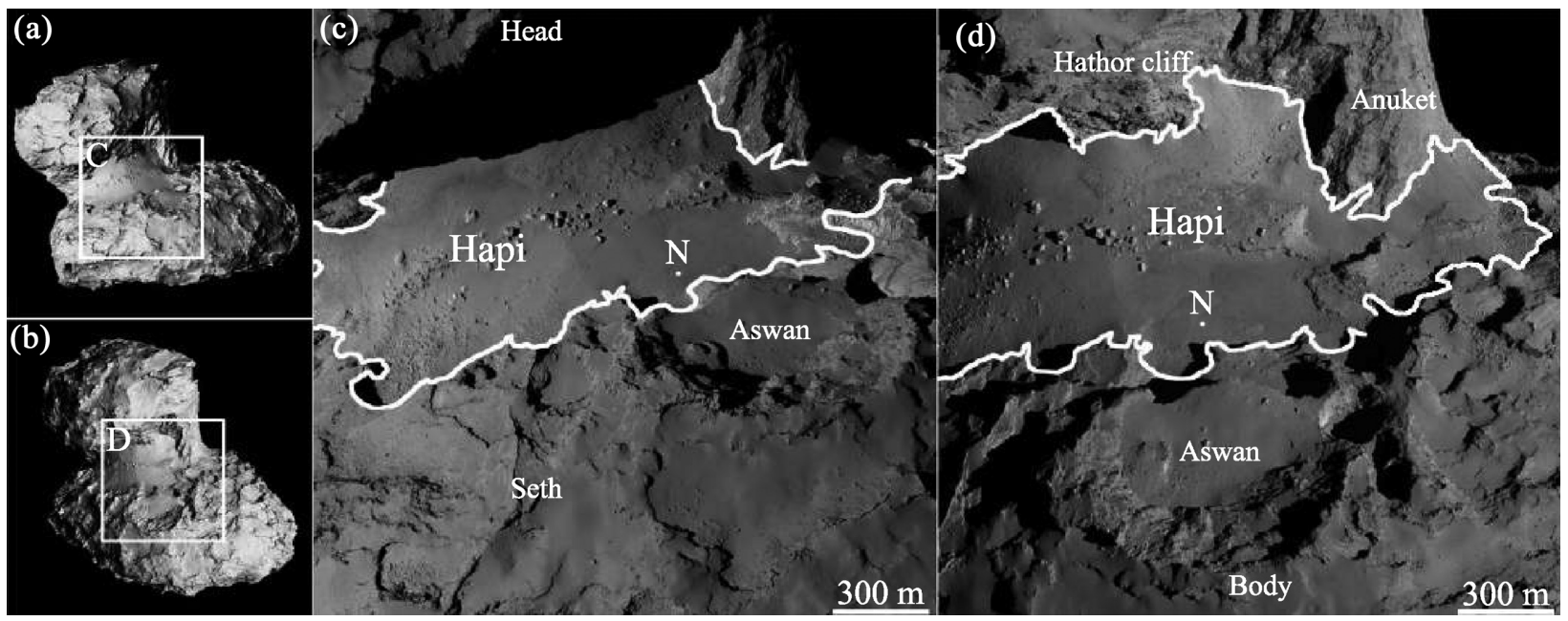

Figure 1. (a) and (b): context images showing where images (c) and (d) were taken. (c) and (d): the Hapi region on 67P outlined with a white boundary. The North pole, indicated by 'N', as well as the contiguous physiographic regions of El-Maarry et al. (2015b) are presented.

by their size range: taluses $(2-18 \mathrm{~m})$, gravitational accumulation deposits $(2-30 \mathrm{~m})$ and diamictons (poorly sorted particles). In the central and western parts of Hapi, at the maximum distances from the Hathor and Seth cliffs, the area studied is dominated by fineparticle deposits, consisting of fine particles unresolvable at the NAC resolution. In the middle of Hapi, a peculiar alignment of boulders is located on the fine-particle deposits. The talus unit is characterized by boulders with diameters ranging from $2-18 \mathrm{~m}$ in size within a fine-grained matrix. This unit is mainly located at the base of the Hathor cliff, but is also present below the Seth cliffs. This unit is often interspersed with gravitational accumulation deposits, which are deposits characterized by boulders with diameters ranging from 2-30 m, within a finer matrix, and that are attributed to gravitational collapses coming from the surrounding niches. The outcropping consolidated terrain unit is the only one belonging to the consolidated material class of Giacomini et al. (2016); Lee et al. (2016). This unit is characterized by a rocky-like terrain without cohesive deposits and it is only present on the west side of Hapi, and in particular at the limit with the Anuket and Seth regions. With the exception of a long fracture $(\sim 300 \mathrm{~m})$ that is observable on the talus unit below the Hathor cliff, all linear features of Hapi are located over the outcropping consolidated terrain. One cuesta/terrace located in the central-west part of Hapi borders a diamicton deposit, which is a heterogeneous and poorly sorted deposit of uncertain origin. Another cuesta/terrace appears near the Anuket limit, where strata and fractures are also present.

\subsection{Boulder size-frequency distributions}

Over the entire study area, we counted 4292 boulders with sizes ranging from $2-47 \mathrm{~m}$ (Figs $4 \mathrm{a}$ and $\mathrm{b}$ ). Out of the $\sim 4300$ boulders, 3050 have sizes $\geq 4.0 \mathrm{~m}$. We stress that, due to the widespread presence of fine-particle deposits located on Hapi, boulders here are partially buried, with their true dimensions necessarily biased (this effect has been observed, for example, in the Imhotep region, El-Maarry et al. 2017). Nevertheless, based on the specific location inside Hapi, the smallest sizes should be more affected by such a blanket than the largest ones, likely leaving the derived size-frequency distributions $\geq 4.0 \mathrm{~m}$ meaningful.
The Hapi area is delimited with a white boundary in Fig. 1 and it is $1.18 \mathrm{~km}^{2}$ wide. We plotted the resulting cumulative size-frequency distribution (SFD) per $\mathrm{km}^{2}$ and derived a power-law index of -2.5 $+0.1 /-0.2$ in the size range $4-20 \mathrm{~m}$ (black continuous line in Fig. 4b). The only previous power-law index computed on the neck of 67P was derived by Pajola et al. (2015) on boulders $\geq 7 \mathrm{~m}$ and it was $-2.2+/-0.2$, comparable with the value presented here. None the less, if from our new boulders statistics we extract the power-law index from the same size range $(7-20 \mathrm{~m})$ we derive a power-law index of $-2.3+0.1 /-0.2$ (red dashed line in Fig. 4b) that is consistent with the previous result of Pajola et al. (2015). In this work the absolute number of $7 \mathrm{~m}$ boulders is 255 , comparable to the previously identified value (213). The slight difference between the two may be entirely due to the difference in spatial scale of the images used, allowing here a better identification of the boulders. Nevertheless, the density of boulders $\geq 7 \mathrm{~m}$ per $\mathrm{km}^{2}$ is 216, 3 times larger than the one obtained in Pajola et al. (2015), where 69 boulders $\geq 7 \mathrm{~m}$ per $\mathrm{km}^{2}$ were identified. This difference is only due to the fact that in the previous analysis the value of the area exclusively attributed to Hapi was, instead, coming from the full neck region of $67 \mathrm{P}\left(3.1 \mathrm{~km}^{2}\right)$. This consequently reduced the previous density of $7 \mathrm{~m}$ boulders by the identified factor of $\sim 3$.

Following the same approach used in Pajola et al. (2015) and Pajola et al. (2017c), and given the wide statistics of boulders that we have on Hapi, we decided to derive the boulder SFD from localized areas that are characterized by homogeneous geological settings. We then made use of the geological map presented in Fig. 3 and selected four different talus deposits: one located at the boundary between Seth and Hapi (called Talus 1), two located below the Hathor cliff (called Taluses 2 and 3) and one at the Anuket limit (called Talus 4); see Fig. 5. It is challenging to properly extract the surface area for such irregular areas on the Digital Terrain Model (DTM), and therefore we were unable to obtain the cumulative number of boulders per $\mathrm{km}^{2}$. Nevertheless, this does not affect the possibility of identifying the power-law indices of all selections, which are $-4.4+0.5 /-0.9$ for Talus $1,-4.4+0.4 /-0.6$ for Talus $2,-4.2+0.4 /-0.5$ for Talus 3 and $-4.4+0.5 /-0.4$ for Talus 4. Since these power-law indices are all very similar, they might indicate a similar origin. In particular, a -3.5 to -4.5 power-law index falls inside the type-2 ranges presented in Pajola et al. (2016d), consistent with the formation through gravitational events triggered 

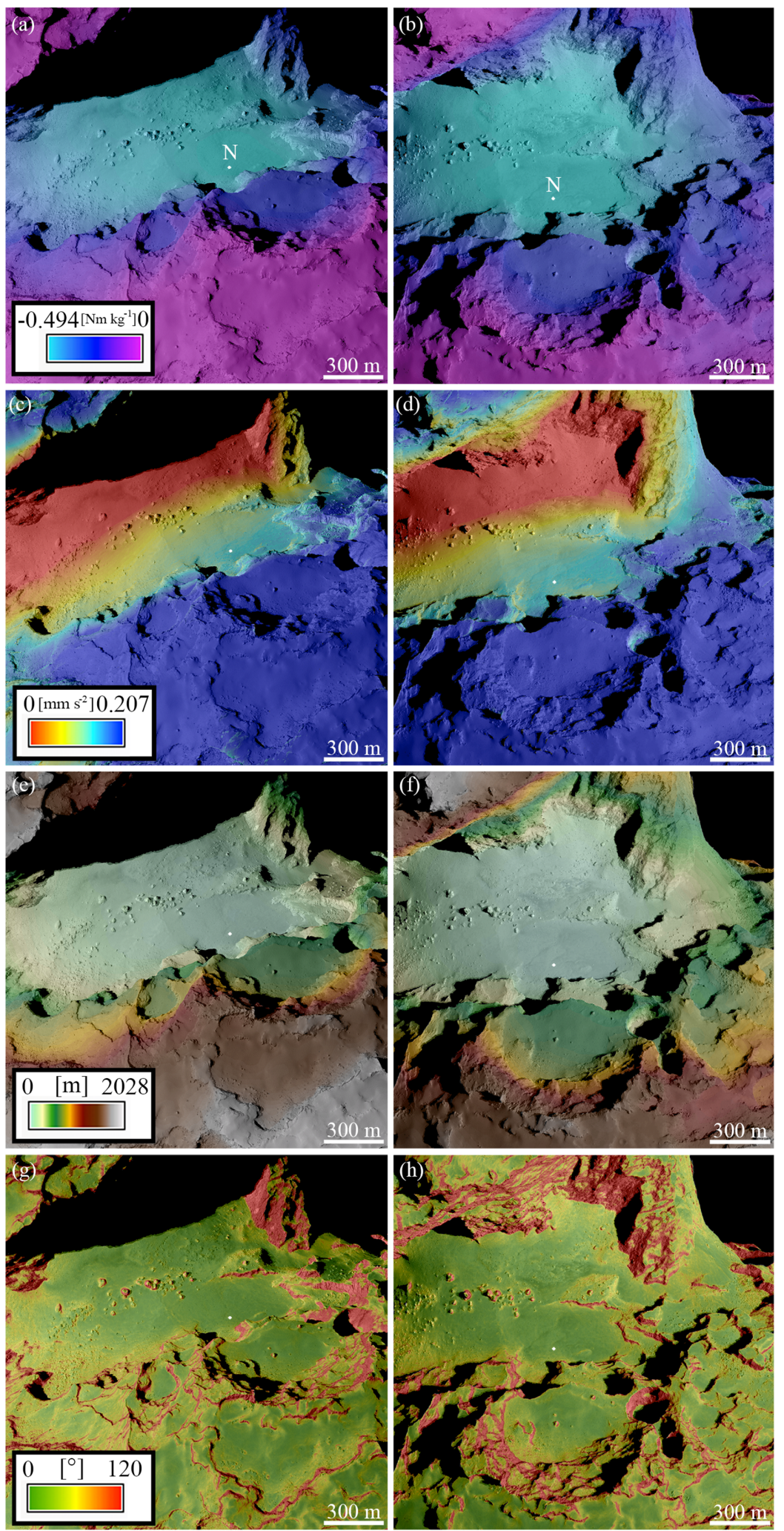

Figure 2. (a), (b) Gravitational potential map of Hapi. (c), (d): Effective gravity map. (e), (f): Dynamic height map. (g), (h): Gravitational slope map. The location of the North pole is indicated with a white dot. 


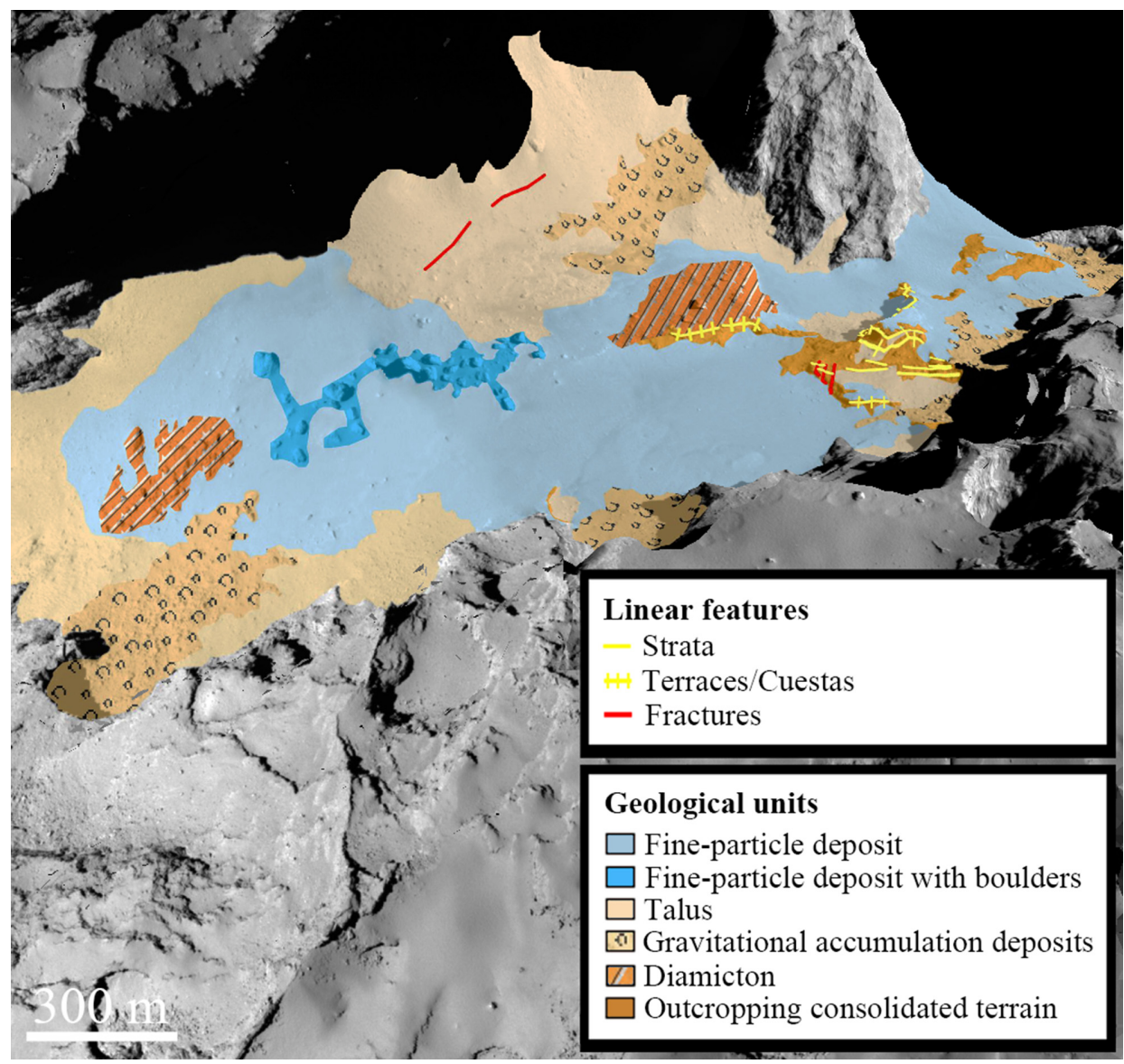

Figure 3. Geological map of Hapi. The linear features and geological units are indicated.

by sublimation and/or thermal fracturing causing regressive erosion of the cliff.

We then derived the boulder SFD from two gravitational accumulation deposits. The first one is located in proximity to Seth; it is called gravitational accumulation deposit 1 and it is characterized by a power-law index of $-3.5+0.3 /-0.5$ (see Fig. $6 a$ ). The second one, called gravitational accumulation deposit 2 , is located below the Anuket boundary and has a power-law index of $-3.0+0.3 /-0.5$ (see Fig. 6b). The geological map supports the interpretation that both deposits are related to cliff collapses: in the first case the power-law index falls inside the type-2 ranges indicated in Pajola et al. (2016d).

In contrast, for the second deposit we observe a shallower powerlaw index due to a decrease of small boulder sizes with respect to the big ones. The reason for this behaviour could be due to the fact that this deposit may have formed earlier than the first one, and active sublimation processes observed on Hapi (Sierks et al. 2015; Keller et al. 2015b) could have had more time to degrade its constituents, making the smaller boulders disappear and hence shallowing the power-law index. Such an evolutionary process is suggested in Pajola et al. (2015, 2016d) in order to explain the more degraded distal deposits on 67P and it could be here represented as a transitional example from a boulder type 2 to a boulder type 3 (Pajola et al. 2015). For the case of the diamicton deposit (Fig. 6c), the $-1.9+0.2 /-0.2$ power-law index falls inside the type- 3 range of Pajola et al. (2016d). It may be representative of a more processed material formed during a gravitational collapse event where continuous sublimation occurred over a prolonged period, hence making the smaller sizes disap- pear with a consequent lowering of the corresponding power-law index.

Such progressive evolution is confirmed by studying the fineparticle deposit with boulders located in the middle of the Hapi region, where big boulders with sizes reaching $40 \mathrm{~m}$ are located (Fig. 6d). The boulder size-frequency distribution computed in the size range $4-16 \mathrm{~m}$ is $-0.6 \pm 0.1$. This shows that the smallest sizes have almost disappeared, hence resulting in an even shallower power-law index than the diamicton one.

\subsection{Layering observations on Hapi}

The Penasa et al. (2017) 3D layered model was used to explain the layered structure of $67 \mathrm{P}$ over multiple areas, located in both the Northern and Southern hemispheres. The model can be used to predict the orientation of the layers in our study area. Fig. 7 shows the traces of predicted layers over terrains with a gravitational slopes greater than $35^{\circ}$, hence slopes that are wiped clear of debris and where the layering is more visible. The predicted orientation is consistent with the terraces located on the west side of Hapi, previously indicated in the geological map (Fig. 3). This suggests that these are indeed structurally related to the inner layering of the body.

The Hapi region is located at the junction between the comet's two lobes. Therefore, by exploiting the 3D layered model of Penasa et al. (2017) and the observed structural continuity of the outcropping terrains, it is possible to establish the pertinence of Hapi either to the head or to the body. Fig. 8(a) shows a map of the terrains that exhibit structural continuity with the body and head. Although Hapi 


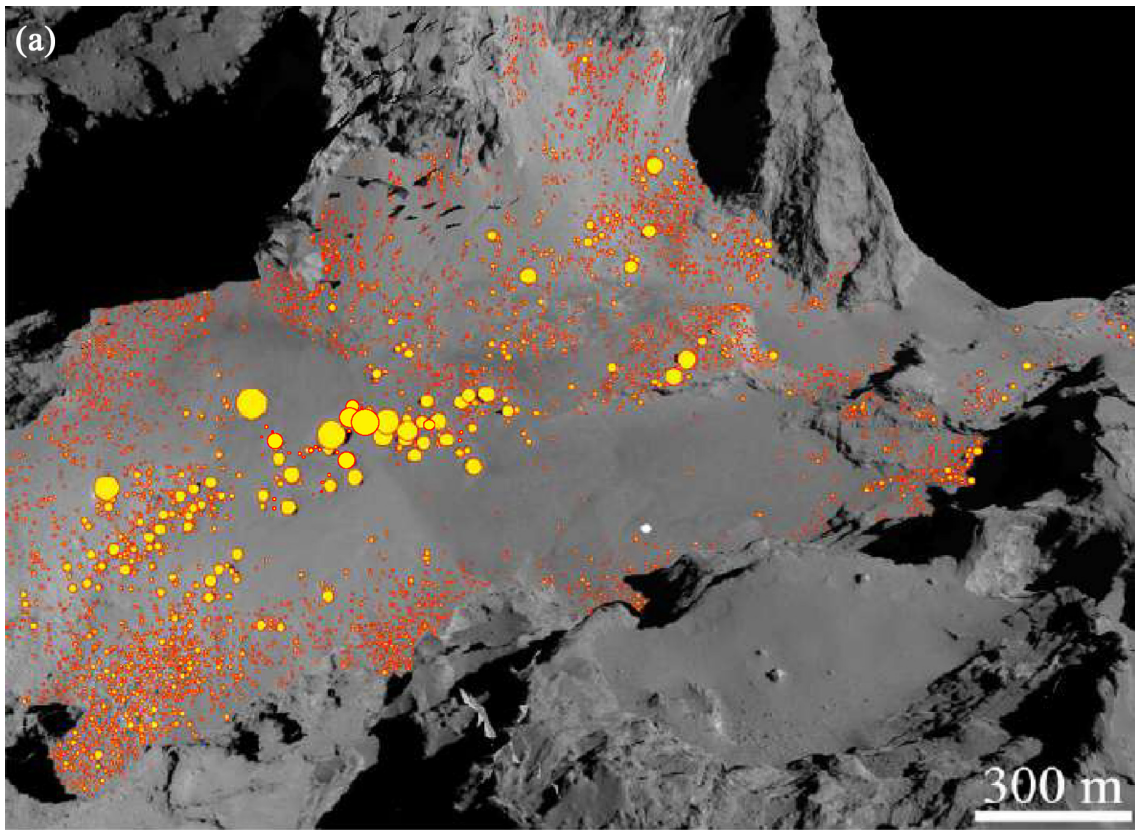

(b)

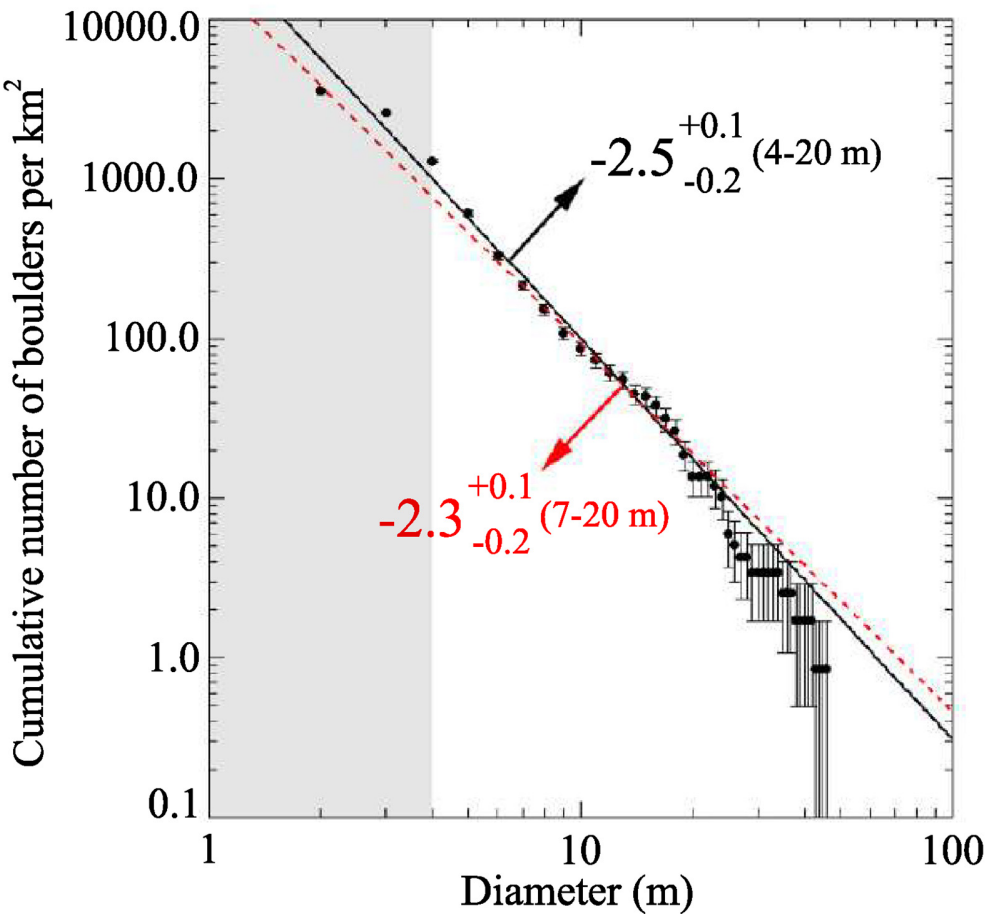

Figure 4. (a) Spatial distribution of the boulders identified in the Hapi region. (b) Cumulative number of boulders per km ${ }^{2}$ and corresponding power-law indices derived from the 4-20 m (black continuous line) and from the 7-20 m size ranges (red dashed line). The total area of the Hapi region (its boundary is indicated in Fig. 1) extracted from the 3D shape model is $1.18 \mathrm{~km}^{2}$. Both the continuous black and red dashed lines are fitted regression lines to the data. The bin size is $1 \mathrm{~m}$.

is largely covered by dusty deposits that cannot be unambiguously associated with either lobe, it is still possible to establish a separation between the two. As pointed out in the geological map description, in the central part of Hapi there is a peculiar alignment of boulderlike features, reminiscent of a ridge, that may represent the remnants of a past cliff layer. Such features seem to entirely cross the region from end-to-end as well as appearing to have the same concavity as the layers of the body. This suggests that they might have been formed by the in-place fragmentation of outcropping, cliff-layered materials pertinent to the body of 67P, that are now mostly overlaid by the dust cover (Penasa et al. 2017).

By perpendicularly cross-cutting the 3D layered model on Hapi, it is possible to see that the Seth layers gently dip towards the neck. In particular, the body's layers are predicted to be dipping at $\sim 40^{\circ}$ beneath the dust cover of Hapi, where the aligned boulder-like features appear, with an orientation compatible with the terraces on top of the Aswan cliff (Pajola et al. 2017a); see Fig. 8(b). 

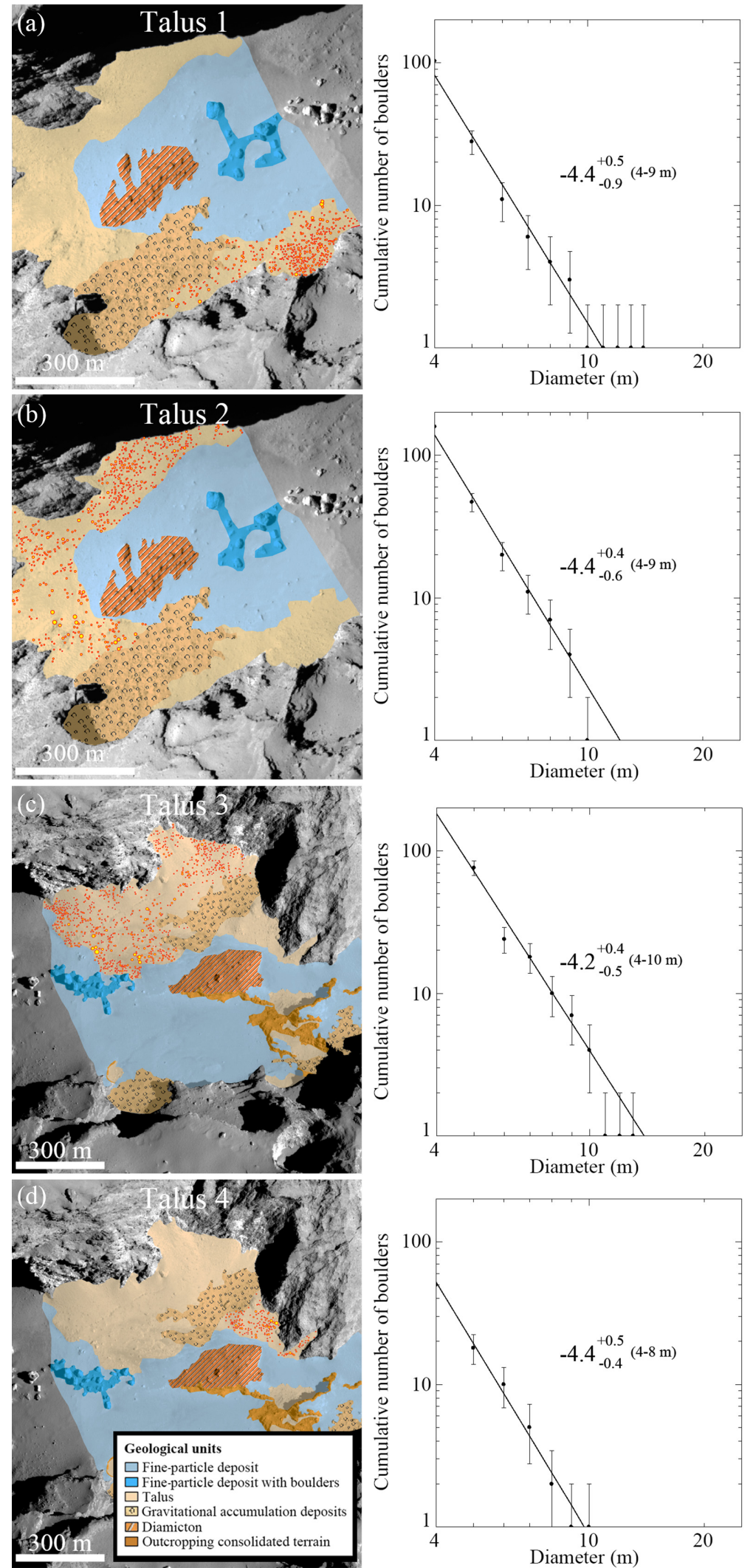

Figure 5. Boulder SFD computed on four different taluses located on Hapi. In the corresponding plots both the power-law indices and the size ranges used for the computation are indicated. 

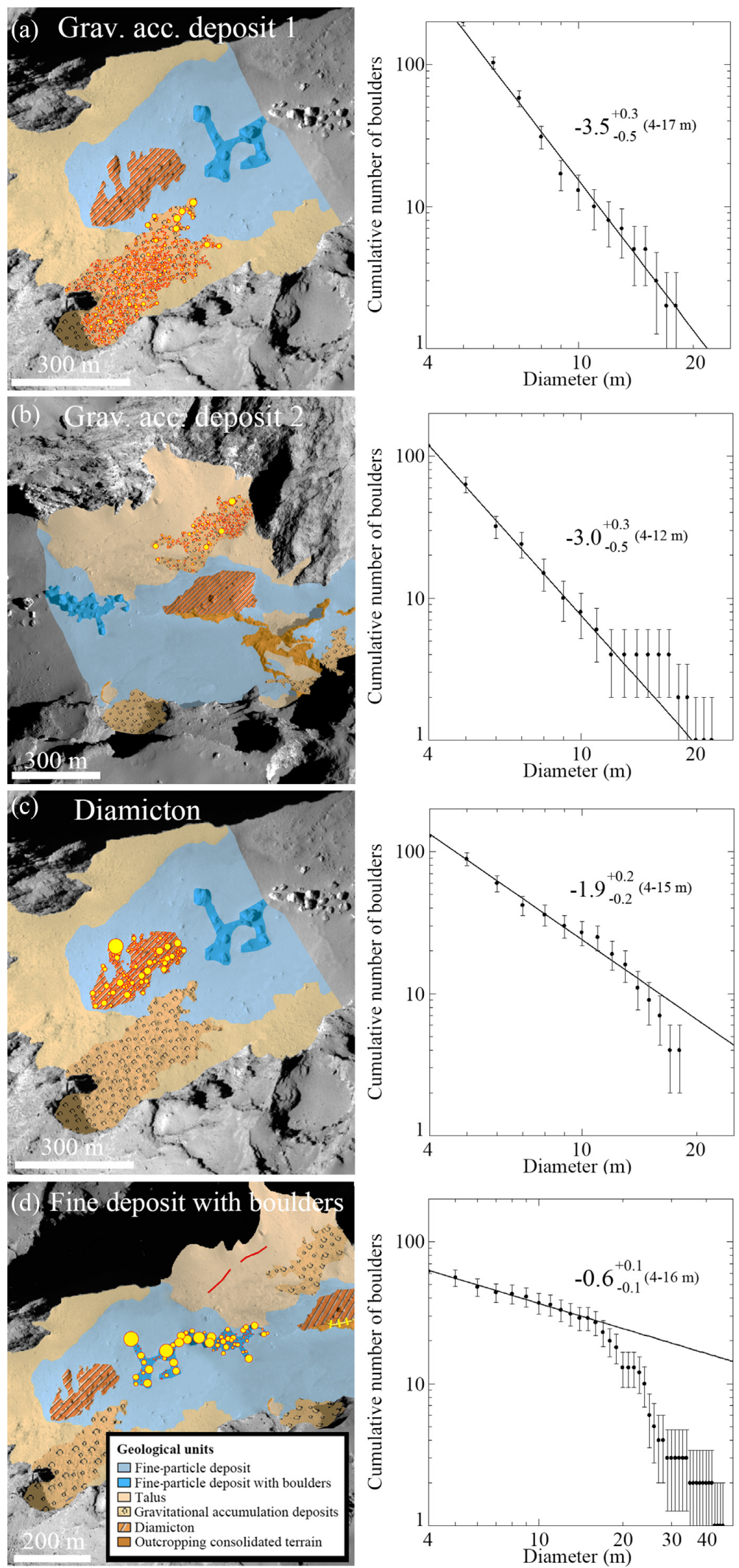

Figure 6. Boulder SFD computed on two gravitational accumulation deposits, one diamicton deposit and on the fine-particle deposit with boulders. In the corresponding plots both the power-law indices and the size ranges used for the computation are indicated. 


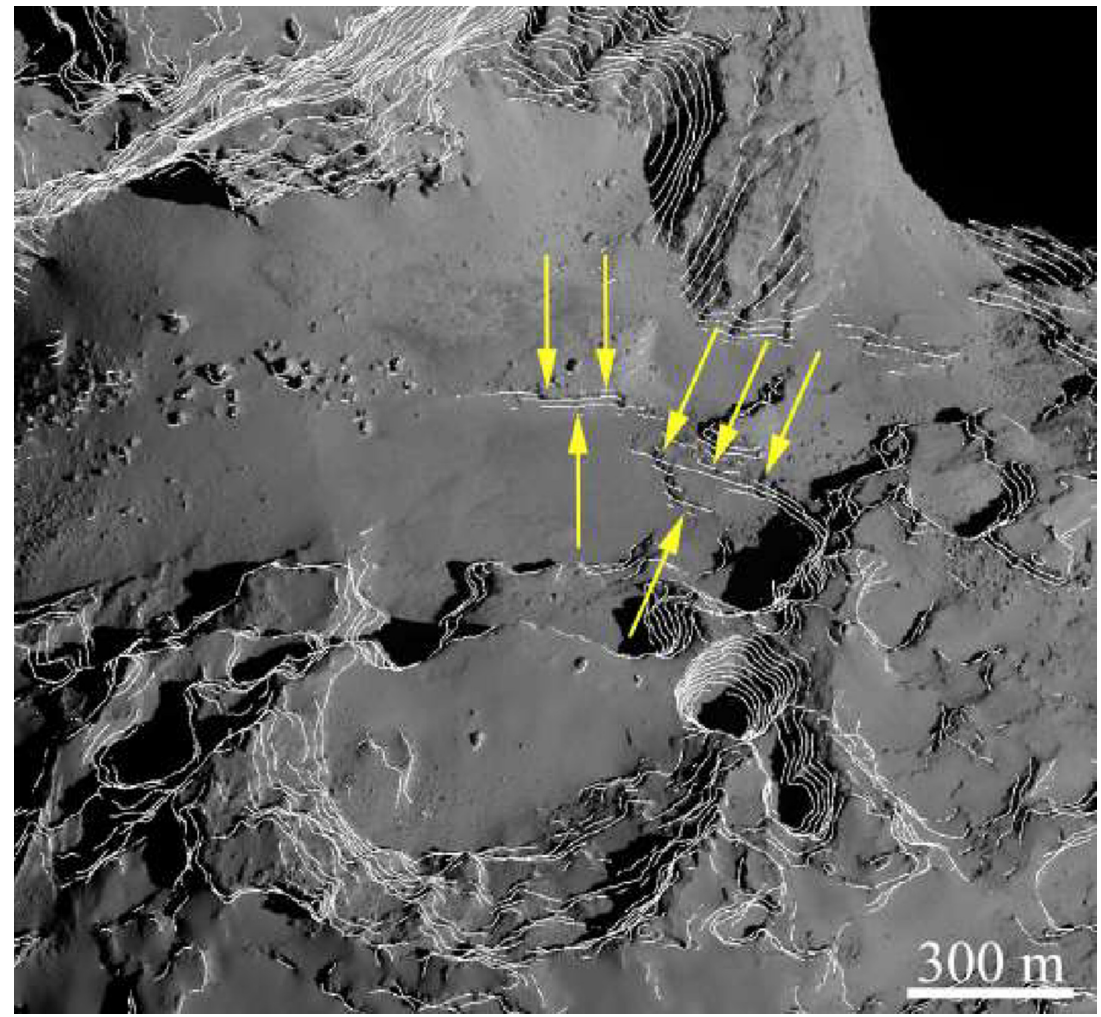

Figure 7. The modelled orientation of the layer traces present on the body and head of 67P identified with white lines. The terraces (indicated with yellow arrows) outcropping at the border of Hapi are well explained by the 3D layered model of Penasa et al. (2017), and are consistent with a structural continuity of the rest of the layering on the body.
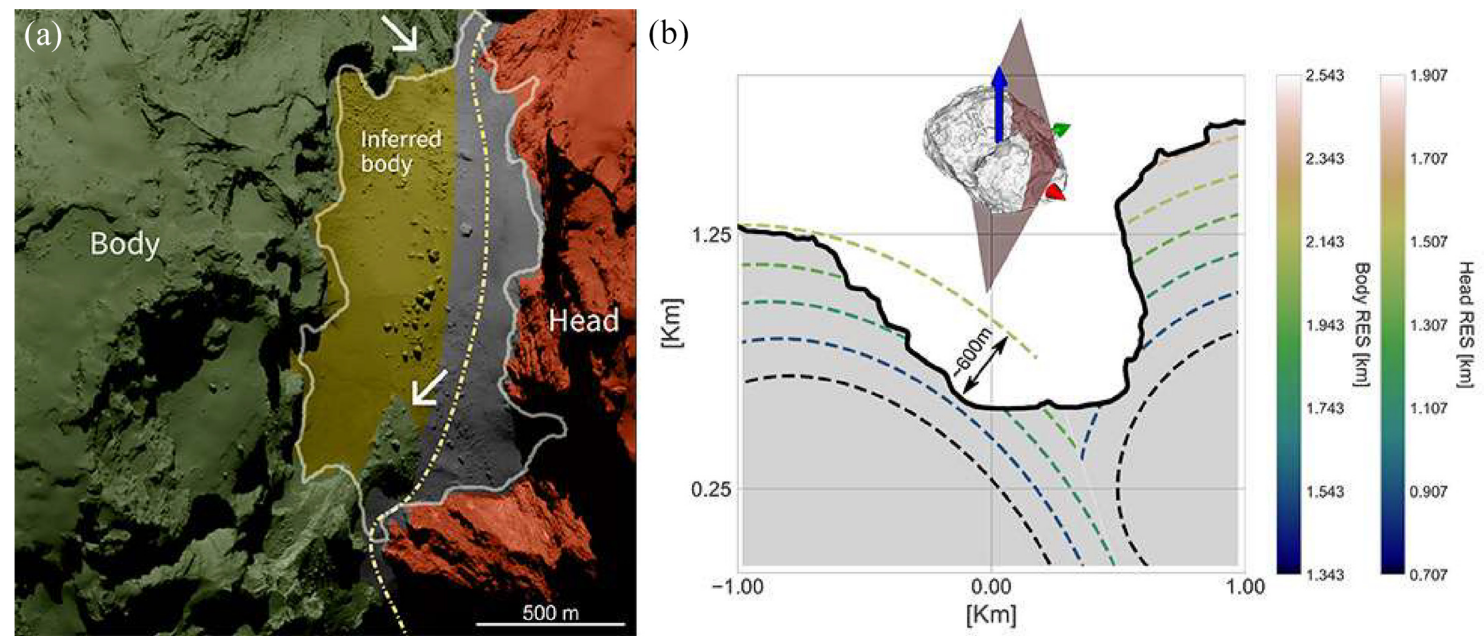

Figure 8. (a) The possible boundary between the body and the head of 67P, as inferred by comparing the 3D model of Penasa et al. (2017) with the outcropping terrains exhibiting a structural continuity with either lobe. The yellow dashed line represents a possible line of separation between the two lobes, but it must be considered an approximation due to the presence of the dust cover that cannot be unambiguously associated with either lobe. (b) Cross-section perpendicular to Hapi, and aligned with the gravity vector at the centre of Hapi. This illustrates the predicted layer orientation in the surrounding area, as computed by the 3D model of Penasa et al. (2017). The colour bars indicate the reference-ellipsoid semimajor axes (RES) for both the head and the body as in Penasa et al. (2017).

\subsection{Colours and spectrophotometry}

Fig. 9 shows the spectral slopes of the Hapi region and its neighbouring regions as observed by the Rosetta mission in 2014 August. The spectral slopes of Hapi are typically 2-3 per cent $100 \mathrm{~nm}^{-1}$ bluer than the surrounding regions, as shown by Fornasier et al. (2015); in addition, they are relatively uniform, showing only subtle variations
(Sierks et al. 2015; Fornasier et al. 2015). Hapi belongs to the group having the lowest spectral slope between the different regions of the comet (Fornasier et al. 2015). The central part of Hapi, where the big boulders are present (see Figs 3, 4, 5 and 6 for context) has 0.5 percent $100 \mathrm{~nm}^{-1}$ lower spectral slopes than the rest of Hapi (Fig. 9). Contrastingly, the regions closer to the Aswan and Hathor cliffs are peppered with material having higher spectral slopes. A 


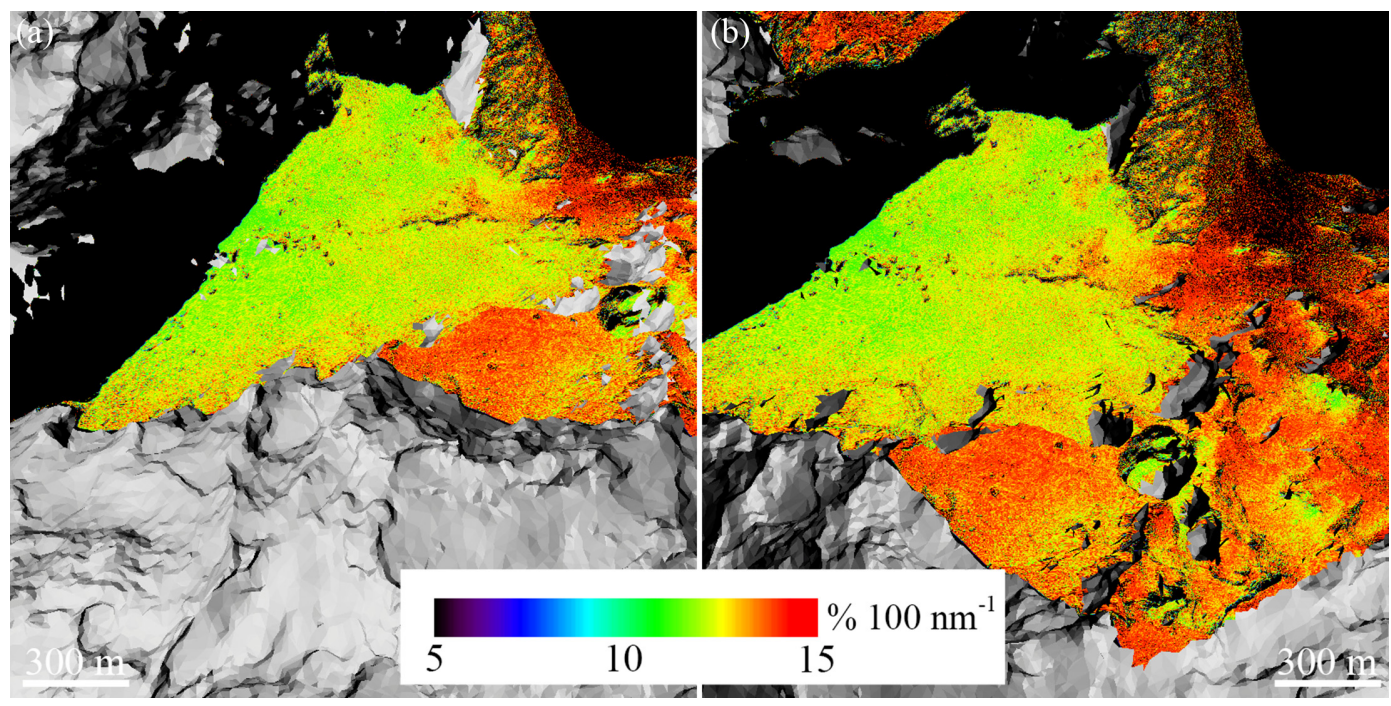

Figure 9. Spectral slope maps of Hapi computed on the 2014 August 22 images (Table 1) and reprojected with the same view of Figs 1(c) and (d).

similar pattern was reported in Sierks et al. (2015) with colour maps showing very subtle colour variation inside Hapi.

Despite the ROIs being chosen to cover each different geological unit (see Figs 3 and 10a for the geological context), the mean spectra of each selected ROI all have similar increasing values with wavelength. There are two weak spectral features detectable at $480 \mathrm{~nm}$ and $701 \mathrm{~nm}^{6}$ (Fig. 10b). Some ROIs are clearly covered with slightly darker or brighter material than others (Fig. 10b). The main difference between the mean spectra of the ROIs is the significantly redder spectrum of ROI 10 (Fig. 10c), which is the one located on the Aswan plateau (Pajola et al. 2016d). Moreover, Hapi, as for the rest of the comet, shows important phase reddening effects. However, its spectral slope between different phase angles (Fornasier et al. 2015, 2016) is always among the smallest measured for comet $67 \mathrm{P}$.

Using the same ROIs, we calculated the mean spectral slope values given in Table 2. As also seen in the relative reflectance, all ROIs have very similar spectral properties. Therefore, the mean spectral slope values show very subtle variations. Only ROI 10 (the Aswan terrace) has a noticeably redder value than the other ROIs.

\subsection{Thermal behaviour of Hapi}

In order to study the thermal behaviour of the Hapi region and to evaluate how both the surface temperatures and the absorbed flux changed during a cometary day, we applied both Models B and C of Keller et al. (2015b) on the study area. ${ }^{7}$ Moreover, to show how the thermal behaviour is so peculiar on Hapi, we compared the obtained results with four other locations situated on different regions on 67P, two on the head, called Hatmehit and Ma' at, and two on the body, called Imhotep and Ash (Fig. 11a).

The first model, called Model B (Keller et al. 2015b), describes the comet's surface as being made of a $50 \mu \mathrm{m}$ layer of dust above the ice/dust mixture of the nucleus, just about the minimum to

${ }^{6} \mathrm{The} 701 \mathrm{~nm}$ emission is attributed to the coma between the spacecraft and the nucleus, and potentially attributed to $\mathrm{H}_{2} \mathrm{O}^{+}$and $\mathrm{NH}_{2}$ (Fornasier et al. 2015, fig. 12 and discussion therein).

${ }^{7}$ The timeframe considered for this computation is 2014 August 29. make the ice invisible to spectroscopy. The computed temperature changes occurring during a comet rotation on the five selected locations are presented in Fig. 11(b). In the Hatmehit, Imhotep, Ash and Ma' at regions there is only one temperature increase and one temperature decrease per day. The difference between these four regions is that for both the Hatmehit and Imhotep case studies the temperature generally requires two to three hours in order to reach its maximum, while it takes five to six hours for the Ash and Ma'at cases. By contrast, the Hapi region is affected by four abrupt changes during each cometary rotation. This is strictly related to its specific location, where mutual shadows are repeatedly cast by the head and the body of 67P different times a day. On Hapi, each temperature increase or decrease generally occurs in less than $20 \mathrm{~min}$. The same temperature trend occurs by applying Model $\mathrm{C}$ (Keller et al. 2015b), which is a model that describes the comet's surface as being made of a $1 \mathrm{~mm}$ layer of dust above the ice/dust mixture of the nucleus. The biggest difference to the previous model is the peak temperature value on all regions, with a maximum value reached on Hapi and Hatmehit of $\sim 200 \mathrm{~K}$, and $\sim 220 \mathrm{~K}$ on Ma' at and Ash. The absorbed flux plots of Fig. 11(c) similarly show the multiple abrupt changes that occur only on Hapi. Moreover, the nonzero values of the Hapi curve in Fig. 11(c) highlight the self-heating component, not present on the four comparison regions (Fig. 12). In addition, we underline that the facet normals in Hapi are parallel to the spin vector of $67 \mathrm{P}$, hence during a cometary day the illumination of the Sun is characterized by an almost constant incidence angle.

\subsection{Jet activities}

In 2014 July, when Rosetta was still more than $5000 \mathrm{~km}$ away from the nucleus of 67P, imaging data from the OSIRIS cameras had already captured jet-like features originating from the Hapi region (Lara et al. 2015; Sierks et al. 2015). During the following months, as the spacecraft descended to less than $30 \mathrm{~km}$ above the surface of the nucleus, more images were obtained, showing collimated dust jets from this area (Lin et al. 2015). This early dust activity was likely driven by the sublimation of water ice from the top layer of the nucleus, hence showing a repetitive behaviour following the variation of insolation (Gulkis et al. 2015; 

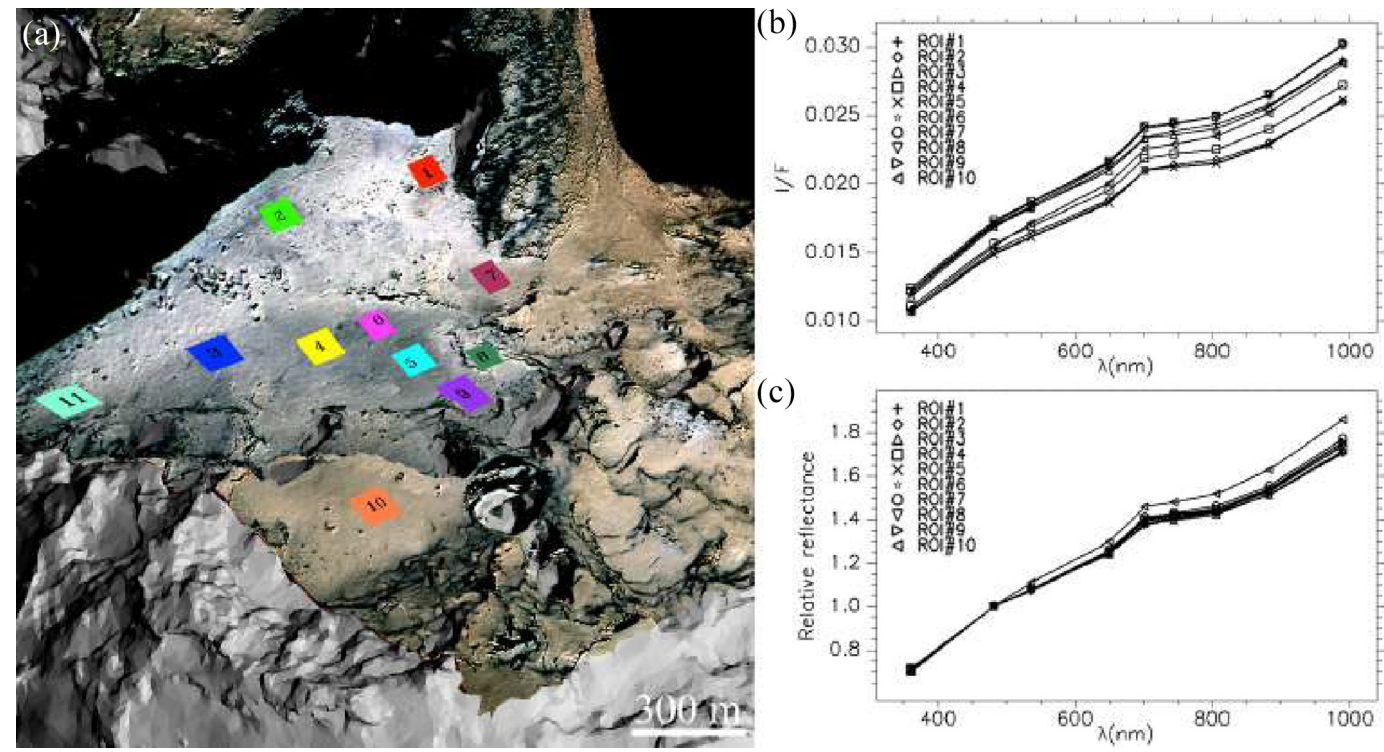

Figure 10. (a): The ROIs selected on Hapi's different geological units (each unit is selected at least once). (b): Mean I/F spectrum for each ROI of (a). (c): Relative reflectance spectra normalized at $480.7 \mathrm{~nm}$. The data acquired on 2014 August 22 (Table 1) are used for the calculations.

Table 2. Mean spectral slopes within the ROIs displayed in Fig. 10(a). As in Table 1 , the phase angle of the observations is $\sim 35^{\circ}$.

\begin{tabular}{lc}
\hline ROI & $\begin{array}{c}\text { Mean spectral slopes } \\
\% 100 \mathrm{~nm}^{-1}\end{array}$ \\
\hline 1 & 11.93 \\
2 & 11.60 \\
3 & 11.58 \\
4 & 12.04 \\
5 & 12.16 \\
6 & 12.10 \\
7 & 12.81 \\
8 & 12.07 \\
9 & 12.29 \\
10 & 13.50 \\
11 & 12.14 \\
\hline
\end{tabular}

Hässig et al. 2015). De Sanctis et al. (2015) found that a water cycle was maintaining the existence of water ice within the diurnal thermal skin depth. By fitting modelled coma properties to measured ones, it was found that Hapi had a higher activity level (at early times pre-perihelion) compared to other regions in the Northern hemisphere of the nucleus (Fougere et al. 2016; Marschall et al. 2016).

The intense gas and dust emission from Hapi forms fine structures in the near-nucleus coma. High-resolution imaging data show jetlike features extending from a few tens to hundreds of metres, varying with both illumination conditions and observing geometries (Lin et al. 2015; Shi et al. 2018). On a large scale, the concave topography of the neck promotes the formation of jets by focusing outgoing gas and dust flows, a mechanism suggested by Crifo et al. (2002). This phenomenon is most obvious in images where the boresight of the camera is aligned with the neck (as in Fig. 13b), where dust flows are projected to form a prominent 'jet'. Further studies with detailed modelling of jets in Hapi show that topography not only performs as a focusing lens but also affects local temperatures and shadowing effects that form the observed jet features (Shi et al. 2018).

\section{DISCUSSION}

As soon as the Rosetta spacecraft approached comet 67P, it was clear that the Hapi region was one of the most intriguing areas of the comet's Northern hemisphere, with its unique location at the transition between the head and the body of 67P (Thomas et al. 2015b), its early jet activity (Shi et al. 2018) and its bright terrains (Fornasier et al. 2015). Such intriguing properties led to suggestions that Hapi be one of the possible landing sites for Philae. Due to the non-compliance of the engineering safety requirements, it was rapidly discarded during the early site selection (Ulamec et al. 2015).

The mass-wasting processes occurring at different times at Hapi's boundaries (see, for example, the Aswan cliff collapse in the Seth region, Pajola et al. 2017a) resulted in the superimposed deposits with different boulder size-frequency distributions that are visible today. This interpretation is supported by the gravitational framework of Hapi, as well as by the power-law values obtained from the boulder size-frequency distributions derived on taluses and gravitational accumulation deposits located on Hapi, mostly falling inside the type- 2 range of Pajola et al. (2015). Instead, the fine-particle deposits located in the central part of Hapi (Fig. 3) are considered to be made of sub-metre-size aggregates coming from the Southern hemisphere during each perihelion passage, and uniformly covering Hapi with an average thickness estimated between 1 and $2 \mathrm{~m}$ (Fulle et al. 2019). Indeed, Keller et al. (2017) suggest that during the northern winter, when Hapi is not illuminated, active particles ejected from the active Southern hemisphere are trapped in the gravitational minimum of Hapi, back-falling on this area characterized by absent cometary activity. Nevertheless, due to Hapi's topography and local gravity field, the thickness of these perihelion deposits may be significantly thinner than the fine-particle deposits in the diamictons, gravitational accumulation deposits and similar units. In this case, the inbound erosion due to 
(a)

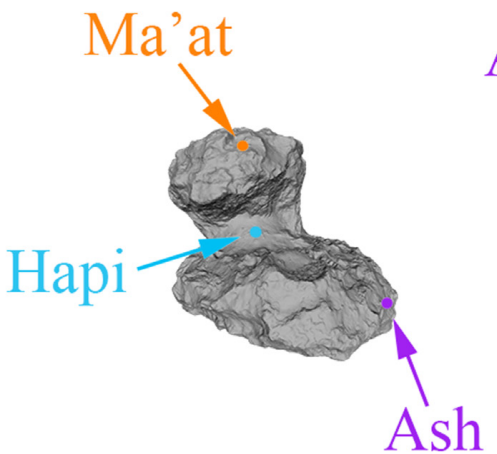

(b)
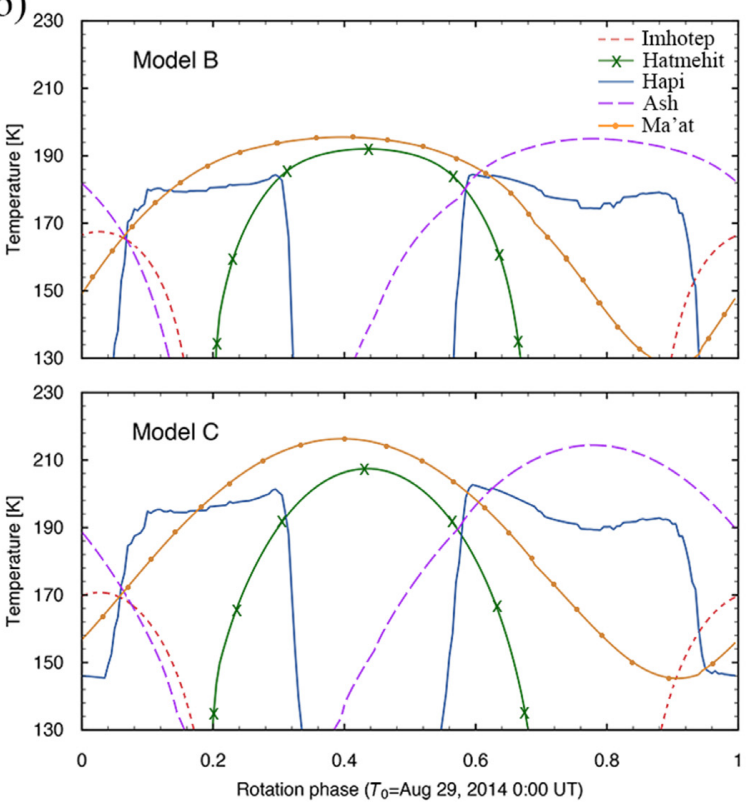

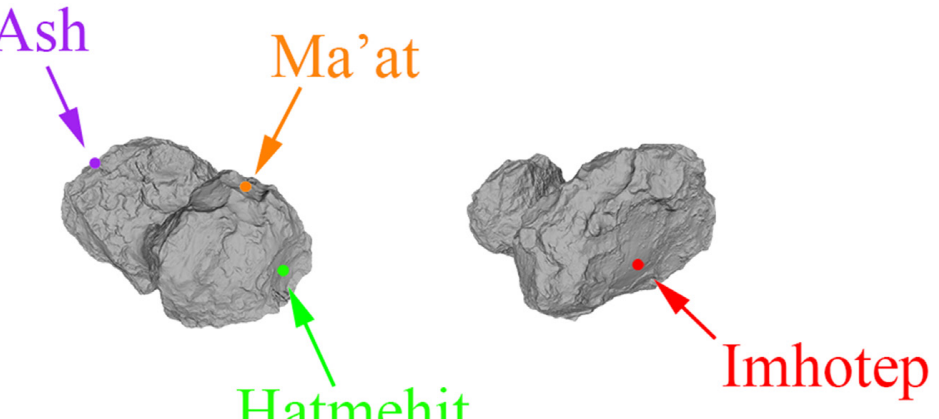

(c)
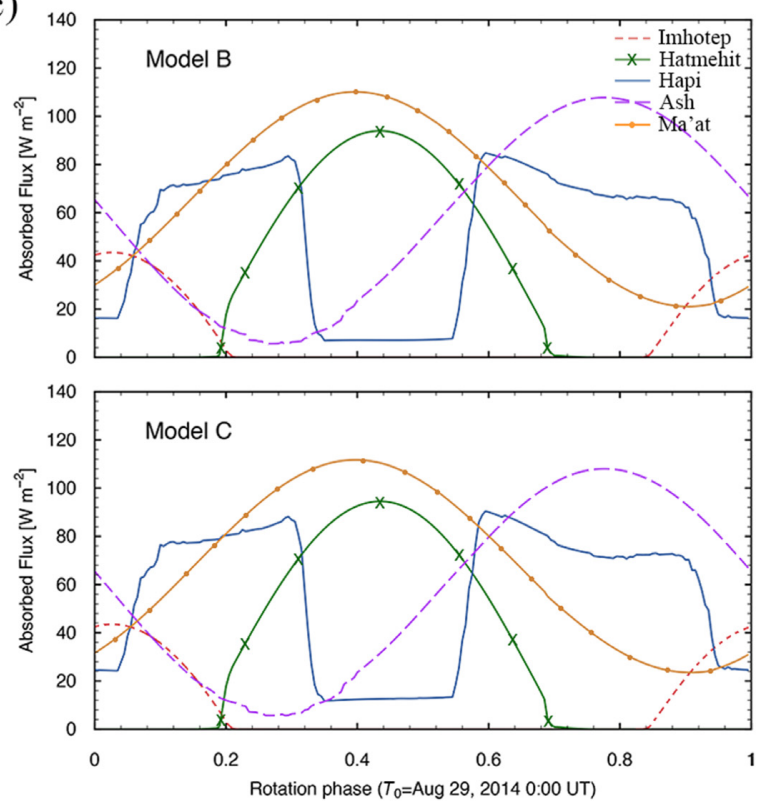

Figure 11. (a) The locations on Hapi, Hatmehit, Imhotep, Ash and Ma'at where we computed the temperature changes during a full rotation of 67P, starting on 2014 August 29, at 0:00 UT (the date and time that we started both the temperature and the absorbed flux is called $T_{0}$ ). (b) The temperature variations on the five locations based on Models B and C of Keller et al. (2015b). The reason for cropping the plots at $130 \mathrm{~K}$ is that whenever there is no illumination, the Keller et al. (2015b) models are not accurate, given that the equilibrium assumption is not verified. For this reason, showing the temperature below $130 \mathrm{~K}$ would be misleading. (c) The absorbed flux on the five locations based on Models B and C of Keller et al. (2015b).

ice sublimation and dust ejection due to 'self-cleaning' (Pajola et al. 2017c; Fulle et al. 2019) reduces both the deposits' thickness and the amount of small-size boulders, resulting in the shallower trend that we observe with the SFD analysis.

The outcropping consolidated terrains located on the western side of Hapi exhibit a clear structural continuity with the layers of the body. This observation is further confirmed when comparing the predicted orientation of the layers (Penasa et al. 2017) and the orientation of the cliffs exposing the consolidated material (Fig. 9a). In Hapi's central part remnants of a possible past cliff layer bulge out of the local average topography (Fig. 9b) in the form of boulder-like features, and present the same curvature of the underlaying layers. Such evidence supports the onion-like structure of the body of 67P suggested in Massironi et al. (2015).

The complexity of the area is demonstrated by the presence of a $\sim 300 \mathrm{~m}$ long fracture running below the Hathor cliff on Hapi's talus unit. This fracture is coupled with the one located in its neighbouring region Anuket, and it is considered to result from activity-induced torques, which are tensile stresses due to the increase or decrease in the spin rate of 67P (Keller et al. 2015a). Indeed, finite-element models (Hirabayashi et al. 2016) suggest that increases in the spin rate could lead to the development of tensile stresses in the neck region (Hapi and Anuket), with the formation of new fractures and the reactivation of pre-existing ones.

The dusty blanket located on Hapi covers an underlying topography that could be represented, from a textural point of view, by the Southern hemisphere side of the comet's neck, called Sobek (El-Maarry et al. 2016). This area is primarily constituted by outcropping consolidated terrains, where strata, terraces as well as scarps, are visible but are highly fractured, resulting in a rough surface texture (Lee et al. 2016). Unlike Hapi, the Sobek terrains are clearly exposed because this is the side of the comet that is highly active when 67P reaches its perihelion. This results in a maximum surface activity that blows off any possible dusty blanket that could settle there.

Hapi's surface is brighter and bluer than 67P's average surface (Fornasier et al. 2015), and comparisons of its spectral properties with active water-ice-rich areas and with laboratory mixtures characterized by water ice showed that it is indeed enriched in water ice (Oklay et al. 2016). This was independently confirmed through 


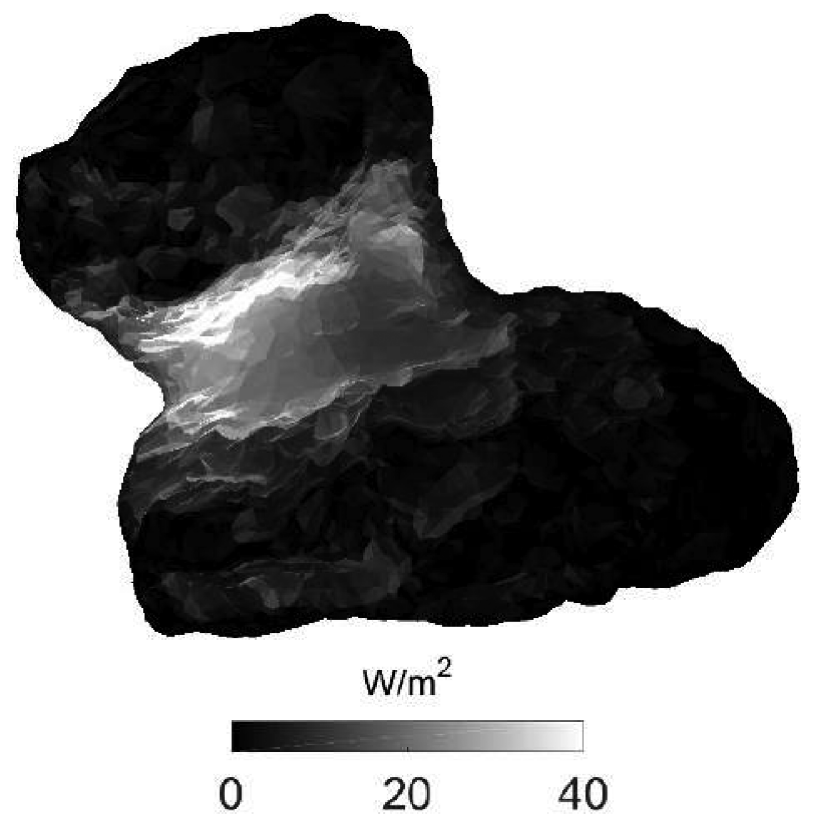

Figure 12. Increase of energy input over the nucleus due to the effect of self-heating computed as from Hu et al. 2017 for 2014 August 29 at 15:43 UT.
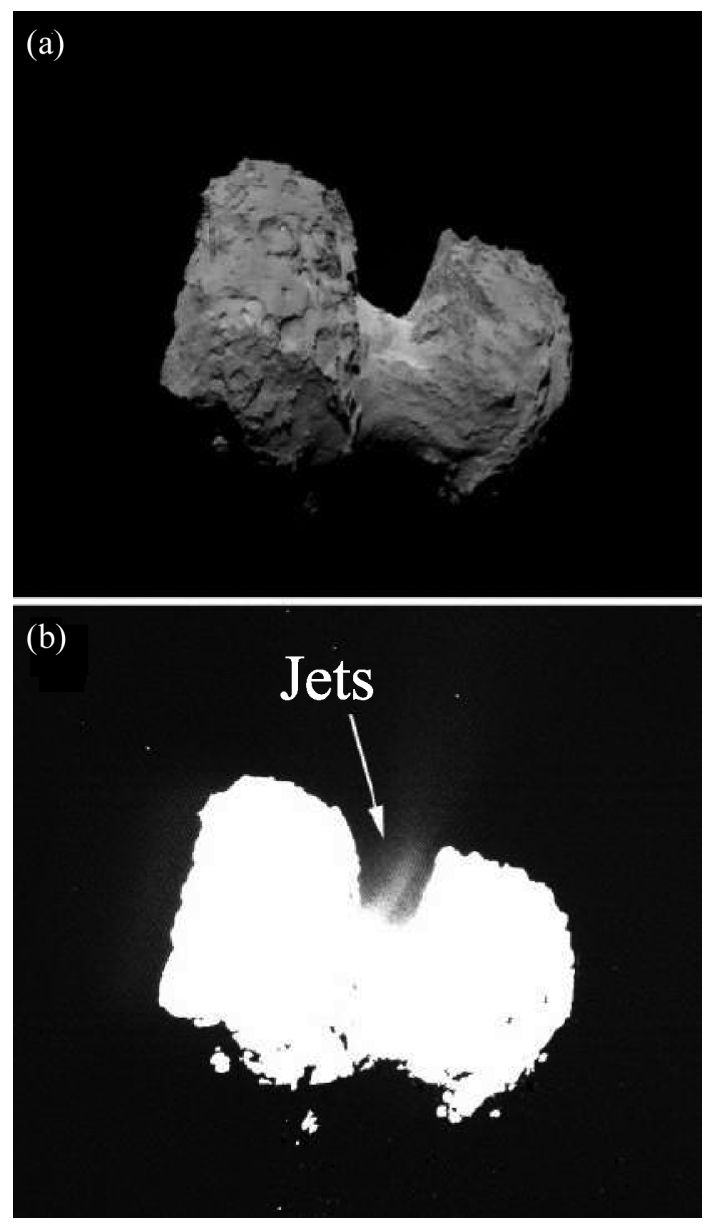

Figure 13. (a) OSIRIS-NAC image taken on 2014 August 3, at a distance of $377 \mathrm{~km}$ from comet $67 \mathrm{P}$. In this image the boresight of the camera is aligned with the neck of 67P. The corresponding scale is $7.06 \mathrm{~m} \mathrm{pixel}^{-1}$. The image was taken when 67P was at 3.61 au from the Sun. (b) Same image as in (a) but stretched to show the jet activity coming from Hapi. spectroscopic observations performed by Filacchione et al. (2016), and De Sanctis et al. (2015) that reported in Hapi the presence of water ice close to shadows. They interpreted this as evidence of the diurnal cycle of water ice, later observed also in other regions close to perihelion (Fornasier et al. 2016). Despite the presence of water ice, the spectral variegation within Hapi is subtle, and even though there are specific darker/brighter terrains, such as those identified in the talus below the Hathor cliff, there is no significant detectable difference in their spectral profiles and the differences between units are all minor. This is either due to the blanketing effect caused by the dust coming from the south (Keller et al. 2017; Lai et al. 2016), hence hiding the possible surface variegation, or to the fact that spectral slopes alone are not sensitive enough to detect variegation. The latter requires investigation of the region in smaller areas with sophisticated methods, which is beyond the scope of this study. However, the first possibility is true for Hapi's surroundings (Oklay et al. 2016)

As indicated by the thermophysical analysis, in the 2014 August timeframe Hapi was characterized by extremely fast temperature and illumination changes, with in addition a self-heating effect occurring largely in the neck of 67P. Such phenomena have been directly connected to the early activity that was observed on 67P, where Hapi was in shadow over about half of the spin period (in the 2014 August-December timeframe) and, when illuminated, it underwent the fastest temperature variations on the comet's surface. The ice-rich aggregates coming from the Southern hemisphere and deposited on Hapi's surface during the last perihelion passage were prone to sublimate right as the comet emerged from aphelion, hence producing the activity observed in 2014 August (Keller et al. 2017). In addition, such fast temperature changes favoured a thermal cracking rate, directly connected to erosion and hence surface activity (Alí-Lagoa, Delbo' \& Libourel 2015).

Hapi's special location and relatively strong water activity provide unique conditions for investigating the development of cometary jets. Observed jet-like features in OSIRIS imaging data are indeed projections of three-dimensional structures of the nearnucleus dust coma. Though they appear to be highly variable, these features result from a combination of local topography and observation geometry (Shi et al. 2018). Since a large portion of 67P's Northern hemisphere is dust-deposited like Hapi, the same mechanism could be ubiquitous, forming the nominal diurnal jet features before perihelion.

\section{CONCLUSIONS}

By using the OSIRIS-NAC imagery data set taken in 2014 August, we performed a multidisciplinary analysis of the Hapi neck region, located on 67P's Northern hemisphere. We provided a gravitational framework for the area, in addition to a geomorphological map to identify the different terrain units in which boulder size-frequency distributions were derived. The power-law indices found from the analysis support the interpretation that both taluses and accumulation deposits are the result of multiple cliff collapses occurring at different times, while the fine-particle deposits, mainly located in the central part of Hapi, are considered to be made of submetre aggregates coming from the Southern hemisphere during each perihelion passage. A different origin can be suggested for both the consolidated terrains on the western part of Hapi and the centrally aligned boulder-like features reminiscent of a ridge. Indeed, they present a structural continuity with layers of the $67 \mathrm{P}$ body supporting its onion-like structure, while the central ridge could be formed by the in-place fragmentation of outcropping cliff- 
layered material pertinent to the body of 67P. We also performed a spectrophotometric analysis on different geological units, finding that the variegation of Hapi is relatively subtle. Indeed, there are no significant spectral differences between units, even if specific darker/brighter terrains, such as the ones observed below the Hathor cliff, are present. This is due to the blanketing effect caused by the dust coming from the south that hides a possible variegation of the surface. Nevertheless, the presence on Hapi of water-ice-rich components that are repeatedly and rapidly illuminated, undergoing the fastest temperature changes on the comet's surface, resulted in the strong jet activity of the region observed in 2014 August.

\section{ACKNOWLEDGEMENTS}

We thank the anonymous referee for important and constructive comments that lead to a substantial improvement in the paper. OSIRIS was built by a consortium of the Max-Planck-Institut für Sonnensystemforschung in Göttingen, Germany, CISAS-University of Padova, Italy, the Laboratoire d'Astrophysique de Marseille, France, the Instituto de Astrofisica de Andalucia, CSIC, Granada, Spain, the Research and Scientific Support Department of the European Space Agency, Noordwijk, The Netherlands, the Instituto Nacional de Tecnica Aeroespacial, Madrid, Spain, the Universidad Politechnica de Madrid, Spain, the Department of Physics and Astronomy of Uppsala University, Sweden, and the Institut für Datentechnik und Kommunikationsnetze der Technischen Universität Braunschweig, Germany. The support of the national funding agencies of Germany (DLR), Italy (ASI), France (CNES), Spain (MEC), Sweden (SNSB), and the ESA Technical Directorate is gratefully acknowledged. We thank the ESA teams at ESAC, ESOC and ESTEC for their work in support of the Rosetta mission.

We made use of the ARCGIS 10.2 software and IDL to perform the presented analysis, as well as the SHAPEVIEWER software, available at www.comet-toolbox.com.

\section{REFERENCES}

Alí-Lagoa V., Delbo’ M., Libourel G., 2015, ApJ, 810, L22

Anderson J. A., Sides S. C., Soltesz D. L., Sucharski T. L., Becker K. J., 2004, in Mackwell S., Stansbery E., eds, Modernization of the integrated software for imagers and spectrometers, Technical Report Vol. 35, Lunar and Planetary Inst., Houston, Texas, USA

Auger A.-T. et al., 2015, A\&A, 583, A35

Birch S. P. D. et al., 2017, MNRAS, 469, S50

Crifo J.-F., Rodionov A. V., Szegö K., Fulle M., 2002, Earth Moon Planets, 90, 227

De Sanctis M. C. et al., 2015, Nature, 525, 500

El-Maarry M. R. et al., 2015a, Geophys. Res. Lett., 42, 5170

El-Maarry M. R. et al., 2015b, A\&A, 583, A26

El-Maarry M. R. et al., 2016, A\&A, 593, A110

El-Maarry M. R. et al., 2017, Science, 355, 1392

Filacchione G. et al., 2016, Icarus, 274, 334

Fornasier S. et al., 2015, A\&A, 583, A30

Fornasier S. et al., 2016, Science, 354, 1566

Fougere N. et al., 2016, A\&A, 588, A134

Fulle M. et al., 2019, MNRAS, 482, 3326

Giacomini L. et al., 2016, MNRAS, 462, S352

Gulkis S. et al., 2015, Science, 347

Hirabayashi M. et al., 2016, Nature, 534, 352

Hapke B., 1993, Theory of reflectance and emittance spectroscopy, Cambridge Univ. Press, New York

Hässig M. et al., 2015, Science, 347, aaa0276

Hu X. et al., 2017, MNRAS, 469, S295
Keller H. U. et al., 2007, Space Sci. Rev., 128, 433

Keller H. U., Mottola S., Skorov Y., Jorda L., 2015a, A\&A, 579, L5

Keller H. U. et al., 2015b, A\&A, 583, A34

Keller H. U. et al., 2017, MNRAS, 469, S357

La Forgia F. et al., 2015, A\&A, 583, A41

Lai I.-L. et al., 2016, MNRAS, 462, S533

Lara L. M. et al., 2015, A\&A, 583, A9

Lee J.-C. et al., 2016, MNRAS, 462, S573

Lin Z.-Y. et al., 2015, A\&A, 583, A11

Lucchetti A. et al., 2016, A\&A, 585, L1

Lucchetti A. et al., 2017, MNRAS, 469, S238

Marschall R. et al., 2016, A\&A, 589, A90

Massironi M. et al., 2015, Nature, 526, 402

Mazrouei S., Daly M. G., Barnouin O. S., Ernst C. M., DeSouza I., 2014, Icarus, 229, 181

Michikami T. et al., 2008, Earth Planets Space, 60, 13

Oklay N. et al., 2016, A\&A, 586, A80

Pajola M. et al., 2015, A\&A, 583

Pajola M. et al., 2016a, MNRAS, 462, S242

Pajola M. et al., 2016b, A\&A, 585, A85

Pajola M. et al., 2016c, A\&A, 592, L2

Pajola M. et al., 2016d, A\&A, 592, A69

Pajola M. et al., 2017a, Nat. Astron., 1, 0092

Pajola M., Rossato S., Baratti E., Pozzobon R., Quantin C., Carter J., Thollot P., 2017b, Icarus, 296, 73

Pajola M. et al., 2017c, MNRAS, 469, S636

Penasa L. et al., 2017, MNRAS, 469, S741

Pommerol A. et al., 2015, A\&A, 583, A25

Preusker F. et al., 2015, A\&A, 583, A33

Preusker F. et al., 2017, A\&A, 607, L1

Rossi A., Fulchignoni M., 1999, Planet. Space Sci., 47, 873

Shi X. et al., 2018, Nat. Astron.

Sierks H. et al., 2015, Science, 347

Thomas N. et al., 2015a, Science, 347

Thomas N. et al., 2015b, A\&A, 583, A17

Ulamec S. et al., 2015, Acta Astronautica, 107, 79

Vincent J.-B. et al., 2015, Nature, 523, 63

Werner R. A., Scheeres D. J., 1997, Celest. Mech. Dynamical Astron., 65, 313

${ }^{1}$ INAF Osservatorio Astronomico di Padova, Vic. Osservatorio 5, 35122 Padova, Italy

${ }^{2}$ Department of Earth Sciences, National Central University, 32054 ChungLi, Taiwan

${ }^{3}$ Deutsches Zentrum für Luft- und Raumfahrt (DLR), Institut für Planetenforschung, Rutherfordstrasse 2, 12489 Berlin, Germany

${ }^{4}$ Center of Studies and Activities for Space, CISAS, ÀG. Colombo', University of Padova, Via Venezia 15, 35131 Padova, Italy

${ }^{5}$ Max-Planck-Institut für Sonnensystemforschung, Justus-von-Liebig-Weg 3, 37077 Göttingen, Germany

${ }^{6}$ LESIA, Observatoire de Paris, Université PSL, CNRS, Univ. Paris Diderot, Sorbonne Paris Cité, Sorbonne Université, 5 Place J. Janssen, 92195 Meudon Pricipal Cedex, France

${ }^{7}$ Jet Propulsion Laboratory, M/S 183-401, 4800 Oak Grove Drive, Pasadena, CA 91109, USA

${ }^{8}$ INAF Istituto di Astrofisica e Planetologia Spaziali, Via del fosso del cavaliere 100, 00133 Roma, Italy

${ }^{9}$ Geosciences Department, University of Padova, Via G. Gradenigo 6, 35131 Padova, Italy

${ }^{10}$ Department of Physics and Astronomy ÀÄ. Galilei”, University of Padova, Vic. Osservatorio 3, 35122 Padova, Italy

${ }^{11}$ CNR-IFN UOS Padova LUXOR, Via Trasea 7, 35131 Padova, Italy

${ }^{12}$ Institute for Space Science, National Central University, 32054 Chung-Li, Taiwan

${ }^{13}$ Space Science Institute, Macau University of Science and Technology, Avenida Wai Long, Taipa, Macau, China 
${ }^{14}$ Laboratoire Atmosphères, Milieux et Observations Spatiales, CNRS \& Université de Versailles Saint-Quentin-en-Yvelines, 78280 Guyancourt, France

${ }^{15}$ Centro de Astrobiologa, CSIC-INTA, 28850 Torrejon de Ardoz, Madrid, Spain

${ }^{16}$ International Space Science Institute, Hallerstrasse 6, 3012 Bern, Switzerland

${ }^{17}$ Scientific Support Office, European Space Research and Technology Centre/ESA, Keplerlaan 1, Postbus 299, 2201 AZ Noordwijk ZH, The Netherlands

${ }^{18}$ Institute for Geophysics and Extraterrestrial Physics, TU Braunschweig, 38106 Braunschweig, Germany

${ }^{19}$ Physics Department, Auburn University, Allison Laboratory, Auburn, AL 36849, USA
${ }^{20}$ Department of Mechanical Engineering, University of Padova, Via Venezia 1, 35131 Padova, Italy

${ }^{21}$ UNITN, University of Trento, Via Mesiano 77, 38100 Trento, Italy

${ }^{22}$ Laboratory for Atmospheric and Space Physics, University of Colorado, 3665 Discovery Drive, CO 80301, USA

${ }^{23}$ INAF Osservatorio Astronomico di Trieste, Via Tiepolo 11, 34143 Trieste, Italy

${ }^{24}$ Instituto de Astrofisica de Andalucia CSIC, Glorieta de la Astronomia, 18008 Granada, Spain

${ }^{25}$ Observatory of the Hungarian Academy of Sciences, PO Box 67, 1525 Budapest, Hungary

This paper has been typeset from a $\mathrm{T}_{\mathrm{E}} \mathrm{X} / \mathrm{LT}_{\mathrm{E}} \mathrm{X}$ file prepared by the author. 